\title{
VARIATIONAL SUMS FOR ADDITIVE PROCESSES
}

\author{
WILLIAM N. HUDSON AND J. DAVID MASON
}

Abstract. Let $X(t), 0 \leqq t \leqq T$, be an additive process, and let $X_{n k}$ be the $k$ th increment of $X(t)$ associated with the partition $\Pi_{n}$ of $[0, T]$. Assume $\left\|\Pi_{n}\right\| \rightarrow 0$. Let $\beta$ be the Blumenthal-Getoor index of $X(T)$ and let $2 \geqq \gamma$ $>\beta$. When the partitions are nested, $\Sigma_{k}\left|X_{n k}\right|^{\gamma}$ converges a.s. to $\sum\left\{|J(s)|^{\gamma}\right.$ : $0 \leqq s \leqq T\}$, where $J(s)$ is the jump of $X(t)$ at $s$. This convergence also holds when the partitions are not nested provided either $X(t)$ has stationary increments or $1 \geqq \gamma>\beta$. This extends a result of P. W. Millar and completes a result of S. M. Berman.

1. Introduction. This note treats stochastic processes with independent increments and which are continuous in probability. Let $X(t), 0 \leqq t \leqq T$, be a version with right continuous paths, with left limits at each point, and with a countable number of (mobile) discontinuities. Assume $X(t)$ has no Gaussian component. The presence of a Gaussian component would force the sums of interest here to diverge, except for the case of squared variation. The characteristic function of $X(t)$ is

$$
E \exp (i u X(t))=\exp \left\{i u \alpha(t)+\int_{-\infty}^{\infty}\left(e^{i u x}-1-\frac{i u x}{1+x^{2}}\right) \nu_{t}(d x)\right\},
$$

where $\nu_{t}$ is the Levy measure of $X(t)$. We assume $\alpha(t)$ is of bounded variation. Associated with $\nu_{T}$ is the Blumenthal-Getoor index

$$
\beta \equiv \beta_{T}=\inf \left\{\delta \geqq 0: \int_{-1}^{1}|x|^{\delta} \nu_{T}(d x)<\infty\right\} .
$$

When $\beta<1$ we assume (1) becomes $\exp \left\{\int_{-\infty}^{\infty}\left(e^{i u x}-1\right) \nu_{t}(d x)\right\}$.

Define $J(s) \equiv X(s)-X(s-)$ to be the jump of $X(t)$ at $s$. For each Borel set $A, \nu_{T}(A)$ is the expected number of jumps of $X(t)$ in $[0, T]$ of size in $A$. For $\Pi_{\mu}=\left\{t_{\mu, 0}, \ldots, t_{\mu, m(\mu)}\right\}, 0=t_{\mu, 0}<t_{\mu, 1}<\cdots<t_{\mu, m(\mu)}=T$, a partition of $[0$, $T$ ], set $X_{\mu, k} \equiv X\left(t_{\mu, k}\right)-X\left(t_{\mu, k-1}\right), \quad k=1, \ldots, m(\mu)$.

In [1], Berman stated the following result.

Let $\Pi_{n}$ be a nested sequence of partitions of $[0, T]$ such that $\left\|\Pi_{n}\right\|$ $=\max _{k}\left|t_{n, k}-t_{n, k-1}\right| \rightarrow 0$. Then for $2 \geqq \gamma>\beta$,

$$
\lim _{n \rightarrow \infty} \sum_{k}\left|X_{n k}\right|^{\gamma}=\sum\left\{|J(s)|^{\gamma}: 0 \leqq s \leqq T\right\} \text { a.s. }
$$

In a personal communication, P. L. Brockett pointed out a gap in equation

Received by the editors January 21, 1975.

AMS (MOS) subject classifications (1970). Primary 60F05, 60G99.

Key words and phrases. Variational sums, infinitely divisible distributions, stochastic processes with independent increments. 
(5.11) [1, p. 242] in Berman's proof of Theorem 5.1 which arises from using the Fatou-Lebesgue Lemma for limit supremum. Berman's proof is valid in the case $X(t)$ is symmetric, but, in general, he lacks an upper bound for the variational sum $\sum_{k}\left|X_{n k}\right|^{\gamma}$ which would permit the use of the Fatou-Lebesgue Lemma (or the Dominated Convergence Theorem).

In order to close this gap we extend a result of Millar [3]. Millar showed that for a process as above which also has stationary increments and for a nested sequence of partitions,

$$
\sup _{n} \sum_{k}\left|X_{n k}\right|^{\gamma}<\infty \text { a.s., } \quad 2 \geqq \gamma>\beta .
$$

Using Berman's Lemmas, we remove the assumption of stationary increments. In the symmetric case, Millar obtained almost sure convergence in (3), but in the general case he only obtained convergence in probability. We establish the almost sure convergence of (3) from (4) thus closing the gap in Berman's proof and extending Millar's results. Hudson and Tucker [2] extended (3) using Berman's results. Our theorem justifies this extension.

Munroe [4] showed that for processes with stationary increments,

$$
\sup \sum_{k}\left|X_{\mu, k}\right|^{\gamma}<\infty \text { a.s., } \quad 2 \geqq \gamma>\beta,
$$

where the supremum is over all partitions of $[0, T]$. Our proof shows that (5) implies (3) without assuming the partitions are nested but only that $\left\|\Pi_{n}\right\| \rightarrow 0$.

Our theorem is as follows:

THEOREM. Let $X(t), 0 \leqq t \leqq T$, be as above (i.e. not necessarily with stationary increments). Let $\Pi_{n}$ be a sequence of partitions of $[0, T]$ such that $\left\|\Pi_{n}\right\| \rightarrow 0$ and let $2 \geqq \gamma>\beta$. If the partitions are nested, then

$$
\sup _{n} \sum_{k}\left|X_{n k}\right|^{\gamma}<\infty \text { a.s. }
$$

and

$$
\lim _{n \rightarrow \infty} \sum_{k}\left|X_{n k}\right|^{\gamma}=\sum\left\{|J(s)|^{\gamma}: 0 \leqq s \leqq T\right\}
$$

If $X(t)$ has stationary increments, or if $\beta<\gamma \leqq 1$, then

$$
\sup \sum\left|X_{\mu k}\right|^{\gamma}<\infty \text { a.s. }
$$

where the supremum is taken over all partitions of $[0, T]$ and $(\mathrm{b})$ holds for a not necessarily nested sequence $\Pi_{n}$ of partitions such that $\left\|\Pi_{n}\right\| \rightarrow 0$.

Proof. First suppose $\beta<\gamma \leqq 1$. In this case it is easy. The characteristic function of $X(t)$ is $\exp \left\{\int\left(e^{i u x}-1\right) \nu_{t}(d x)\right\}$, and the Lévy representation for the paths is

$$
X(t)=\sum\{J(x): 0<s \leqq t\}, \quad 0 \leqq t \leqq T .
$$

Let $\Pi_{\mu}=\left\{t_{\mu, 0}, \ldots, t_{\mu, m(\mu)}\right\}$ be a partition of $[0, T]$. Since $|a+b|^{\gamma} \leqq|a|^{\gamma}$ $+|b|^{\gamma}$ for $0 \leqq \gamma \leqq 1$, it follows that 


$$
\begin{aligned}
\sum_{k}\left|X_{n k}\right|^{\gamma} & =\sum_{k}\left|\sum\left\{J(s): t_{n, k-1}<s \leqq t_{n k}\right\}\right|^{\gamma} \\
& \leqq \sum\left\{|J(s)|^{\gamma}: 0<s \leqq T\right\} .
\end{aligned}
$$

But

$$
E\left[\Sigma\left\{|J(s)|^{\gamma}: 0<s \leqq T,|J(s)| \leqq 1\right\}\right]=\int_{[-1,1]}|x|^{\gamma} \nu_{T}(d x)<\infty .
$$

With probability one only a finite number of jumps have size greater than one, hence, the right side of (6) is finite a.s. Therefore,

$$
\sup _{\mu} \sum_{k}\left|X_{\mu, k}\right|^{\gamma}<\infty \text { a.s., } \quad \beta<\gamma \leqq 1,
$$

where the supremum is over all partitions of $[0, T]$. Together with Munroe's result, this proves (c).

Now let $\left\{\Pi_{n}\right\}$ denote a sequence of nested partitions of $[0, T]$ such that $\left\|\Pi_{n}\right\| \rightarrow 0$ as $n \rightarrow \infty$. Millar proves (a) in Theorem 3.1 of [3] for the case that $X(t)$ has stationary increments. However, his proof only uses this assumption twice; both times he wishes to bound $E\left[\sum_{k}\left|X_{n k}\right|^{\gamma}\right]$. But from Lemmas 5.1-5.3 of Berman, we have that

$$
\varlimsup_{n \rightarrow \infty} E\left[\sum_{k}\left|X_{n k}\right|^{\gamma}\right]<\infty, \quad \max (\beta, 1) \leqq \gamma \leqq 2,
$$

and a fortiori,

$$
\sup _{n} E\left[\sum_{k}\left|X_{n k}\right|^{\gamma}\right]<\infty
$$

if $\nu_{T}$ is concentrated on a bounded interval. Thus, if $\nu_{T}$ is concentrated on $[-M, M]$, we have

$$
\sup _{n} \sum_{k}\left|X_{n k}\right|^{\gamma}<\infty \text { a.s., } \quad \max (\beta, 1) \leqq \gamma \leqq 2 .
$$

Let $Y(t)=\sum\{J(s): 0<s<t,|J(s)|>1\}$, and $Z(t)=X(t)-Y(t)$. Then

$$
\sum_{k}\left|X_{n k}\right|^{\gamma}=\sum_{k}\left|Y_{n k}+Z_{n k}\right|^{\gamma} \leqq 2^{\gamma-1} \sum_{k}\left|Y_{n k}\right|^{\gamma}+2^{\gamma-1} \sum_{k}\left|Z_{n k}\right|^{\gamma} .
$$

If $N$ is the number of jumps of absolute value greater than 1 , then $N$ is finite almost surely. From the crude estimate $\left|\sum_{1}^{n} a_{i}\right|^{\gamma} \leqq 2^{n-1} \sum_{1}^{n}\left|a_{i}\right|^{\gamma}$, it follows that

$$
\sup _{n} \sum_{k}\left|Y_{n k}\right|^{\gamma} \leqq 2^{N-1} \sum\left\{|J(s)|^{\gamma}: 0<s \leqq T,|J(s)|>1\right\}<\infty \text { a.s. }
$$

By (11), $\sup _{n} \sum_{k}\left|Z_{n k}\right|^{\gamma}<\infty$ a.s. and (12) implies that

$$
\sup \sum_{k}\left|X_{n k}\right|^{\gamma}<\infty \text { a.s. }
$$

Hence, (a) is now proved.

We now suppose that the sequence $\left\{\Pi_{n}\right\}$ is such that $\left\|\Pi_{n}\right\| \rightarrow 0$ and that 


$$
\sup _{n} \sum_{k}\left|X_{n k}\right|^{\gamma}<\infty \text { a.s. }
$$

It remains to show that

$$
\lim _{n \rightarrow \infty} \sum_{k}\left|X_{n k}\right|^{\gamma}=\sum\left\{|J(s)|^{\gamma}: 0 \leqq s \leqq T\right\} \text { a.s. }
$$

Define $I_{n j}(\varepsilon)$ to be 1 if there are no jumps of absolute value greater than $\varepsilon$ in $\left(t_{n, j-1}, t_{n, j}\right]$ and 0 otherwise. In order to prove (15) we will show that

$$
\lim _{\varepsilon \downarrow 0} \varlimsup_{n \rightarrow \infty} \sum_{k}\left|X_{n k}\right|^{\gamma} I_{n k}(\varepsilon)=0 \text { a.s., } \quad \gamma>\beta .
$$

Let

$$
Y^{(\varepsilon)}(t)=X(t)-\sum\{J(s):|J(s)|>\varepsilon, 0<s \leqq t\} .
$$

Note that $\left|X_{n k}\right|^{\gamma} I_{n k}(\varepsilon) \leqq\left|Y_{n k}^{(\varepsilon)}\right|^{\gamma}$, and so to prove (16) it suffices to prove that

$$
\lim _{\varepsilon \downarrow 0} \varlimsup_{n \rightarrow \infty} \sum\left|Y_{n k}^{(\varepsilon)}\right|^{\gamma}=0 \text { a.s., } \quad \gamma>\beta .
$$

Fix $\gamma>\beta$ and choose $\delta \in(\beta, \gamma)$. Since $Y^{(\varepsilon)}(t)$ has right and left limits at each point and no jumps of absolute value greater than $\varepsilon$, a Heine-Borel argument shows that for $n$ sufficiently large,

$$
\sup _{k}\left|Y_{n k}^{(\varepsilon)}\right| \leqq 2 \varepsilon
$$

Hence,

$$
\varlimsup_{n \rightarrow \infty} \sum\left|Y_{n k}^{(\varepsilon)}\right|^{\gamma} \leqq(2 \varepsilon)^{\gamma-\delta} \varlimsup_{n \rightarrow \infty} \sum\left|Y_{n k}^{(\varepsilon)}\right|^{\delta} .
$$

Since

$$
\begin{aligned}
\varlimsup_{n} \sum\left|Y_{n k}^{(\varepsilon)}\right|^{\gamma} & \leqq \sum\left\{|J(s)|^{\gamma}:|J(s)|<1,0<s \leqq T\right\}+\varlimsup_{n} \sum\left|Y_{n k}^{(1)}\right|^{\gamma} \\
& <\infty \text { a.s. }
\end{aligned}
$$

letting $\varepsilon \downarrow 0$ we obtain (18) and hence (16).

Since for all $\varepsilon>0$ there are at most a finite number of jumps of absolute value greater than $\varepsilon$, a Heine-Borel argument shows that

$$
\begin{aligned}
\lim _{n \rightarrow \infty} \sum_{k}\left|X_{n k}\right|^{\gamma}\left(1-I_{n k}(\varepsilon)\right) \\
\quad=\sum\left\{|J(s)|^{\gamma}:|J(s)|>\varepsilon, 0<s \leqq T\right\} \text { a.s. }
\end{aligned}
$$

(Without loss of generality assume $\pm \varepsilon$ to be continuity points of $\nu_{T}$ so with probability 1 no jump has absolute value $\varepsilon$.) Then

$$
\lim _{\varepsilon \downarrow 0} \lim _{n \rightarrow \infty} \sum\left|X_{n k}\right|^{\gamma}\left(1-I_{n k}(\varepsilon)\right) \leqq \lim _{n \rightarrow \infty} \sum_{k}\left|X_{n k}\right|^{\gamma},
$$

and hence from (20), 


$$
\sum\left\{|J(s)|^{\gamma}: 0<s \leqq T\right\} \leqq \underline{\lim _{n}} \sum_{k}\left|X_{n k}\right|^{\gamma}
$$

Also,

$$
\begin{aligned}
\varlimsup_{n \rightarrow \infty} \sum_{k}\left|X_{n k}\right|^{\gamma} \leqq & \lim _{\varepsilon \downarrow 0} \varlimsup_{n \rightarrow \infty} \sum_{k}\left|X_{n k}\right|^{\gamma} I_{n k}(\varepsilon) \\
& +\lim _{\varepsilon \downarrow 0} \varlimsup_{n \rightarrow \infty} \sum_{k}\left|X_{n k}\right|^{\gamma}\left(1-I_{n k}(\varepsilon)\right),
\end{aligned}
$$

and hence by (16) and (20),

$$
\lim _{n \rightarrow \infty} \sum_{k}\left|X_{n k}\right|^{\gamma} \leqq \sum\left\{|J(s)|^{\gamma}: 0<s \leqq T\right\} \text { a.s. }
$$

Part (b) follows from (22) and (24). Q.E.D.

\section{REFERENCES}

1. S. M. Berman, Sign-invariant random variables and stochastic processes with sign-invariant increments, Trans. Amer. Math. Soc. 119 (1965), 216-243. MR 32 \#113.

2. W. N. Hudson and H. G. Tucker, Limit theorems for variational sums, Trans. Amer. Math. Soc. 191 (1974), 405-426.

3. P. W. Millar, Path behavior of processes with stationary independent increments, Z. Wahrscheinlichkeitstheorie und Verw. Gebiete 17 (1971), 53-73. MR 48 \#3130.

4. I. Munroe, On the $\gamma$-variation of processes with stationary independent increments, Ann. Math. Statist. 43 (1972), 1213-1220.

Department of Mathematics, University of Utah, Salt Lake City, Utah 84112 (Current address of J. David Mason)

Current address: (William N. Hudson): Department of Mathematics, Bowling Green State University, Bowling Green, Ohio 43403 